Article

\title{
Waveform and Spectral Characteristics of Supraharmonic Unsymmetrical Conducted EMI of Switched-Mode Power Supplies
}

\author{
Leonardo Sandrolini ${ }^{1}$ (D) and Andrea Mariscotti ${ }^{2, *(D)}$ \\ 1 DEI, Alma Mater Studiorum-University of Bologna, 40136 Bologna, Italy; leonardo.sandrolini@unibo.it \\ 2 DITEN, University of Genova, 16145 Genova, Italy \\ * Correspondence: andrea.mariscotti@unige.it
}

Citation: Sandrolini, L.; Mariscotti, A Waveform and Spectral Characteristics of Supraharmonic Unsymmetrical Conducted EMI of Switched-Mode Power Supplies. Electronics 2022, 11, 591. https:// doi.org/10.3390/electronics11040591 Academic Editors: Gianpaolo Vitale and Bor-Ren Lin

Received: 2 January 2022

Accepted: 7 February 2022

Published: 15 February 2022

Publisher's Note: MDPI stays neutral with regard to jurisdictional claims in published maps and institutional affiliations.

Copyright: (C) 2022 by the authors. Licensee MDPI, Basel, Switzerland. This article is an open access article distributed under the terms and conditions of the Creative Commons Attribution (CC BY) license (https:// creativecommons.org/licenses/by/ $4.0 /)$.

\begin{abstract}
Switched-mode power supplies (SMPSs) are a relevant and widespread source of unsymmetrical conducted emissions, as they feed various electrical appliances and information technology equipment in office and residential contexts and, despite their low power rating, the limits are the same as those of larger equipment. Given the peculiar emission waveform (mixing impulsive components and switching components), assessment should be carried out with care to select parameters that guarantee both the detection of the maximum level of emissions and a high level of repeatability and reproducibility. A range of cases based on six different SMPS products was considered, exploring both time- and frequency-domain characteristics, and identifying the most relevant phenomena and advisable parameters choices. Typical behavior and suitable settings were exemplified with a range of measured emission records: the Fourier time window length and EMI receiver dwell time were considered, as well as the relevance of loading conditions to include in a comprehensive test program. Such records were made available in a linked dataset to allow for replication of the experiments and foster further findings and discussion.
\end{abstract}

Keywords: conducted emissions; electromagnetic compatibility; electromagnetic interference; supraharmonics; switched-mode power supplies; transients

\section{Introduction}

Switched-mode power supplies (SMPSs) are extensively used to supply a wide range of items of information technology, entertainment and audio products, electrical appliances and telecommunications. They are deployed in large numbers within residential and light industrial environments, connected to the low voltage (LV) mains: with their low power rating, they have received little attention so far, but their large number hints that their emission characteristics should be considered in more detail. SMPSs feature various power conversion architectures but share common elements that make their input waveforms look quite similar, distinguishing them from high-frequency content: the front-end input stage is based on a simple single-phase rectifier, providing an internal DC-link voltage characterized by a ripple at twice the mains frequency; the conversion process is based on a two-leg (or four-leg) converter, feeding the high-frequency transformer with an almost symmetrical waveform, swinging between the two extremes of the DC-link voltage and, thus, exhibiting alternate polarity of the pattern of emissions. For a low power rating, harmonic distortion limits might not be applied (see Section 7.1 of [1]) and there would be no need for a power factor correction (PFC) stage in this case; a large amount of conducted emissions and their variability with supply conditions can, in reality, be led back to the PFC stage.

Focusing on unsymmetrical conducted emissions above the usual harmonic interval (conventionally extended up to $2 \mathrm{kHz}$ ), the by-products of the switching process feature 
switching fundamentals of some tens of kilohertz and spread into the hundreds of kilohertz and megahertz ranges. Besides the "classical" RF conducted emissions in the known $0.15-30 \mathrm{MHz}$ frequency interval that is extensively regulated by the IEC, CISPR and CENELEC standards [2,3], in the last decade attention has been turned to include the frequency interval below $150 \mathrm{kHz}$ [4-10]: such an interval (named the "supraharmonics interval") extends sharply from $2 \mathrm{kHz}$, or $9 \mathrm{kHz}$ for some in an attempt to include some CISPR specifications [11], and is, in general, not currently regulated by specific electromagnetic compatibility (EMC) limits. Some standards [12-16] cover the $2-150 \mathrm{kHz}$ interval, but not explicitly SMPSs and other similar switching converters.

The most used methods for spectrum analysis and the assessment of conducted emissions for this frequency range are both frequency- and time-domain based: the former consist substantially of a frequency-domain sweep using a spectrum analyzer or EMI receiver, while the latter may be summarized in Fourier-oriented approaches and improved methods featuring a better control of time and frequency resolution (e.g., wavelet packet decomposition). However, it was demonstrated that SMPS emissions feature specific signal characteristics that deserve some amount of tuning and optimization of the methods in order to get accurate, consistent and reliable results [17-19]. These characteristics can be exemplified as a superposition of medium- and high-frequency components featuring different stationarity and spectrum occupation properties and significant spectrum leakage for the main transient impulsive components of the signal. SMPSs may feature different modulation patterns implemented in different onboard regulator technologies so that the behavior may be quite different in principle, although it is sensible that a broad division into some main classes is attainable.

This work addresses this problem of classifying the behavior of SMPSs as far as conducted emissions are concerned in order to derive general rules for signal processing methods. The intention was to identify common characteristics and behaviors of SMPSconducted emissions to define the spectral characteristics of EMI signals with the objective of showing optimized settings for time- and frequency-domain data, measured by means of a digital sampling oscilloscope and an EMI receiver, respectively. The focus was on the demonstration of some good practices and typical phenomena with a wide range of experimental data, which were also made available in a dataset linked to the paper and whose details are provided in Appendix A.

The work is structured as follows. Section 2 describes the measurement setup and the type of data that are acquired both for the time- and frequency-domain tests; these data for the cases discussed in this work were made publicly available and accessible, as described in Appendix A. Section 3 discusses the two techniques exemplifying the variability of results in both the time and frequency domains, both using the short-time Fourier transform (STFT) (referring to parameters and settings already discussed in a previous work [17]) and direct measurements with the EMI receiver (for which the focus was on the effect of the dwell time). The large amount of data is organized by previewing them in Section 4 for each of the six measured SMPSs and the three loading conditions (no load and $25 \%$ and $90 \%$ of the nominal load), linking them to Appendix A, where the dataset structure is explained. The considered frequency range between $2 \mathrm{kHz}$ and $1 \mathrm{MHz}$ was characterized by supraharmonic disturbance and radiofrequency (RF) components, with a wide range of different behaviors that well represent the mechanisms of emissions.

\section{Selected SMPSs and Measurement Setup}

A comprehensive set of SMPSs was considered and their conducted emissions were measured in the time and frequency domains using the setup described below in Section 2.2. SMPS tests were preceded by considerations of their characteristics and modes of operation, together with some insight into typical converter architectures, which are discussed in Section 2.1. 


\subsection{SMPS Operation and Characteristics}

The tested SMPSs featured a $5 \mathrm{~V}$ or $12 \mathrm{~V}$ DC output (depending on model) and were connected upstream to the LV mains, $230 \mathrm{~V}, 50 \mathrm{~Hz}$. The rated power was limited to a few tens of watts so that the output current was in the order of one or a few amperes. Their characteristics are summarized in Table 1.

Table 1. Characteristics of the tested SMPSs: brand and model, no-load voltage and current delivered to the load at the scheduled $25 \%$ and $90 \%$ load conditions.

\begin{tabular}{ccccc}
\hline Acronym & SMPS & $\mathbf{V}_{\text {no-load }}(\mathbf{V})$ & $\mathbf{I}_{\mathbf{2 5} \text {-1oad }}(\mathbf{A})$ & $\mathbf{I}_{\mathbf{9 0} \% \text {-load }}$ (A) \\
\hline LW & Lacie White $(24 \mathrm{~W})$ & 12.94 & 0.51 & 1.83 \\
LB & Lacie Black $(26.4 \mathrm{~W})$ & 11.75 & 0.47 & 1.72 \\
KT & KTEC $(18 \mathrm{~W})$ & 12.19 & 0.37 & 1.43 \\
SH & Shenzhen Honor $(18 \mathrm{~W})$ & 12.45 & 0.38 & 1.45 \\
BA & Balance $(10 \mathrm{~W})$ & 5.212 & 0.51 & 1.94 \\
KA & Kario $(10 \mathrm{~W})$ & 5.144 & 0.51 & 1.91 \\
\hline
\end{tabular}

It may be observed that, in Table 1 the multiplication of the $\mathrm{V}_{\text {no-load }}$ and $\mathrm{I}_{25 \% \text {-load }}$ columns delivered approximately $26-27 \%$ of the rated load, as the internal voltage drop was limited; at a large current for the $90 \%$ load condition, the actual voltage delivered to the load was, in general, reduced relative to the no-load condition, despite the load voltage regulation capability such that the direct multiplication of $\mathrm{V}_{\text {no-load }}$ and $\mathrm{I}_{90 \% \text {-load }}$ gave a power value above $90 \%$ and sometimes in excess of $100 \%$.

The loading for each model was adjusted by connecting a series-parallel combination of resistors with a metallic heatsink. The slight inductive characteristic of such low-value resistors was not relevant to the determination of conducted emissions measured on the AC mains port by means of a LISN (line impedance stabilization network).

Conversely, a minimum time interval for thermal stabilization was necessary in order to have a stable load level and operating point of the SMPS between successive tests. During the first test, it was evident that the thermal stabilization of the loading resistors was reached in about 15-20 min with a satisfactory accuracy of some percent. Instead, the internal mechanisms of emissions of each SMPS took much longer to stabilize, in the order of 100-120 min for a fraction of decibels of target accuracy on the amplitude of the main spectrum components.

The SMPS architectures were different, as well as the adopted modulation strategies. Typical architectures are exemplified in Table 2, based also on available information from the manufacturers [20,21]. They were used to generate one or more operating voltages from an existing variable input voltage. When the input voltage was $\mathrm{AC}$, they were combined to give a half- or full-bridge converter. The SMPS converters examined in this paper were all information technology equipment (ITE) power supplies and, thus, were all low-power converters $(<150 \mathrm{~W})$.

Table 2. Basic architectures for SMPSs.

\begin{tabular}{ccc}
\hline Power & Switching Topology & Galvanic Isolation \\
\hline Low & Buck (step-down) & No \\
Low & Boost (step-up) & No \\
Low & SEPIC (single-ended primary inductance converter) & No \\
Low & Flyback & Yes \\
High & Forward & Yes \\
High & Push-pull & Yes \\
\hline
\end{tabular}

Switching and regulation methods can be broadly classified in terms of the conducted emissions as "deterministic" and "randomized"; the former category refers to techniques with fixed frequency switching, whereas the latter refers to techniques adopting some kind 
of spread-spectrum technique. This resulted in smaller, but fatter, peaks of emissions with de facto spreading of the energy of the deterministic spectrum of emissions onto adjacent frequency bins while preserving the total EMI energy since there was no attenuation. In other words, the switching frequency set by the clock was manipulated to reduce the peak energy and was distributed to other frequencies and their harmonics. There exist several techniques that allow for a spread spectrum, for example, triangular analog dither, pseudo-random, adaptive random spread spectrum and dual random spread spectrum, each performing better at either low or high frequency [22-25]. In the triangular analog dither, the switching frequency is swept within a limited range of about the nominal value; this technique offers a good performance between $150 \mathrm{kHz}$ and $30 \mathrm{MHz}$, with the resolution bandwidth (RBW) requirement of $9 \mathrm{kHz}$. A drawback of this technique is that it may generate audible noise. For the higher frequency bands, above $30 \mathrm{MHz}$, where the RBW requirement is $120 \mathrm{kHz}$, pseudo-random modulation offers better performance. This consists in changing the switching frequency pseudorandomly at every switching cycle in order to generate a fast modulation that is closer to the $120 \mathrm{kHz}$ RBW. Adaptive random spread spectrum changes the modulation frequency at the end of each ramp with the purpose of reducing the audible tone generated by dithering with a fixed frequency. Dual random spread spectrum improves the high-frequency performance of adaptive random spread spectrum: it minimizes the peak EMI in multiple frequency bands by adding pseudorandom cycle-by-cycle dithering on top of the randomly changing triangular profile.

\subsection{Setup for Time- and Frequency-Domain Measurements}

Conducted emissions were measured at the AC side where the SMPS was connected to the AC mains through an LISN, achieving two objectives: the normalization of the mains impedance, thus ensuring the reproducibility of results in compliance with the EMC standards for RF conducted emissions, and filtering out disturbances originating somewhere in the network and propagating to the $230 \mathrm{~V}$ socket. The LISN is a wellknown 3-port network, with a $\pi$-cell featuring two shunt capacitive branches and one series inductor that implements the desired impedance curve for the main power flow between the AC mains and the equipment under test. The LISN is compliant with CISPR 16-1-2 for V-networks with an impedance in the frequency range $9 \mathrm{kHz}$ to $30 \mathrm{MHz}$ with a maximum deviation of $20 \%$ and is a periodically calibrated piece of equipment. The output signal for the measuring equipment (either a digital sampling oscilloscope or an EMI receiver) is derived from the second capacitive branch, which is indeed an RC branch, where the resistive value is adjusted to the asymptotic value of the impedance curve, and, at the same time, also the input impedance of the measuring instrument. Details of the circuit arrangements and connections are shown in Figure 1. Photos of the tested SMPSs and the experimental setup are shown in Figure 2.

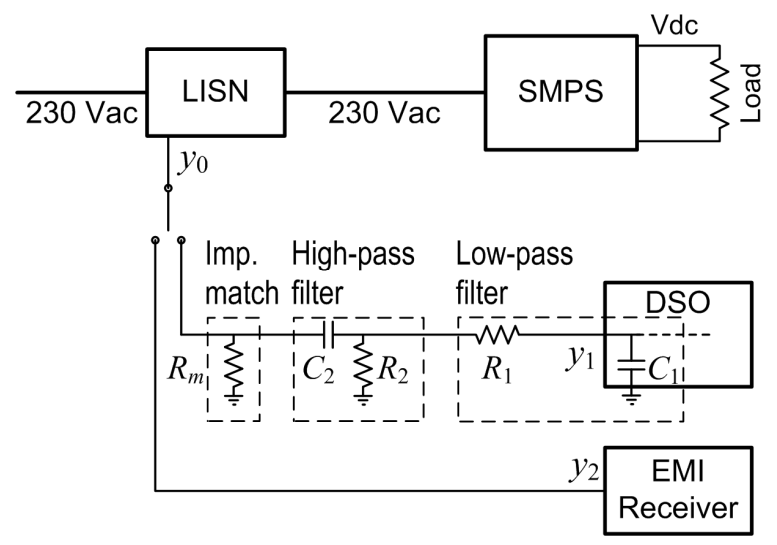

Figure 1. Scheme of the experimental setup: SMPS under test and its DC variable load, LISN (line impedance stabilization network), $50 \Omega$ impedance matching, high-pass filter (1 kHz cutoff) and low-pass filter (2 MHz cutoff), digital storage oscilloscope (DSO) and EMI receiver. 


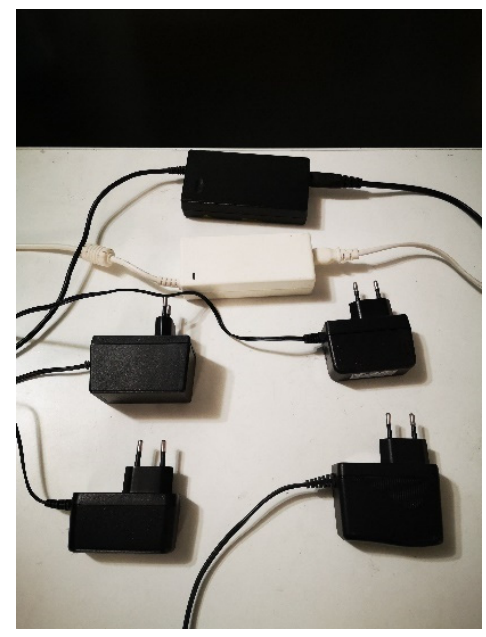

(a)

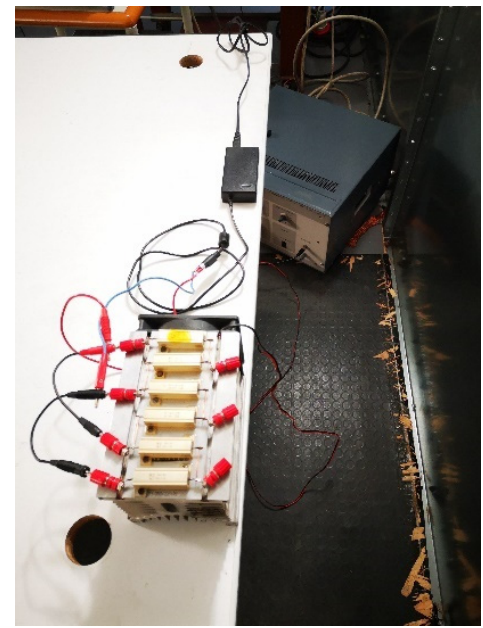

(b)

Figure 2. Photos of (a) the tested SMPSs and (b) the experimental setup.

The LISN output $y_{0}$ was thus connected as follows:

- Either to an 8-bit DSO with a sampling rate of $10 \mathrm{MSa} / \mathrm{s}$ (ensuring that the target frequency interval of $2-150 \mathrm{kHz}$ could be achieved in terms of the sampling time and record length);

- Or to an EMI receiver with a peak detector, followed by max hold and dwell time ranging between 1 and $200 \mathrm{~ms}$.

The DSO measurement chain was equipped with three additional elements:

- A $50 \Omega$ impedance-matching barrel $R_{m}$ to implement the mentioned $50 \Omega$ loading of the capacitive branch of the LISN;

- An anti-aliasing low-pass filter $R_{1} C_{1}$, which was designed starting from a maximum frequency of $1 \mathrm{MHz}$ (positioning its cutoff frequency one octave above at $2 \mathrm{MHz}$, which was very compatible with the said sampling frequency of $10 \mathrm{MHz}$ ) and exploiting the input capacitance of the DSO channel (found to be quite accurate and stable at $15 \mathrm{pF}$ ), thus resulting in a resistive value $R_{1}=1.5 \mathrm{k} \Omega$ (negligible in terms of the voltage drop in series to the $1 \mathrm{M} \Omega$ input resistance of the DSO);

- A high-pass filter $C_{2} R_{2}$ that further attenuated the $50 \mathrm{~Hz}$ mains fundamental and its harmonics and designed around a resistive value large enough not to disturb the impedance matching and to remarkably influence the low-pass filter (set to $5 \mathrm{k} \Omega$ ) and a resulting series capacitance $C_{2}$ with a negligible reactance over the desired frequency interval. The use of a high-pass filter is supported by the suggested increase of the decoupling factor in [26] and confirmed by [27] for similar measurements.

\section{Discussion of the Form of Data and Their Variability}

\subsection{Apparent Characteristics of Time-Domain Waveforms}

In general, impulsive conducted emissions caused by switching events are located at zero crossings of the mains sinusoidal voltage; it was observed that at high load power levels, additional pulses were located with $3.5-4.5 \mathrm{~ms}$ delay, approximately anticipating the maximum of the voltage sinusoidal waveform. The signal $y_{1}$ at the DSO input (qualitatively similar to the signal $y_{0}$, as provided at the LISN output) was an intermittent waveform, deprived of the $50 \mathrm{~Hz}$ mains voltage component and its low-order harmonics, not only as a matter of safety but also to improve the dynamic range. The zero-mean AC-coupled high-pass filtered signal was characterized by a main pulse followed by rapid oscillations, as shown in Figure 3. The instantaneous oscillation frequency was in the order of $20-30 \mathrm{kHz}$ and was in relation to the internal switching frequency; the repetition rate followed the rectified voltage ripple at $100 \mathrm{~Hz}$, twice the mains frequency. 


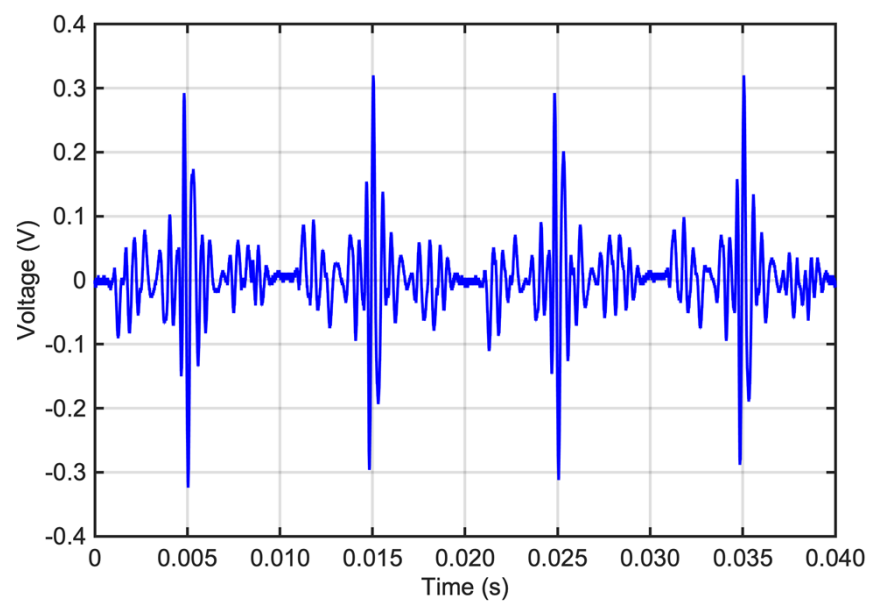

(a)

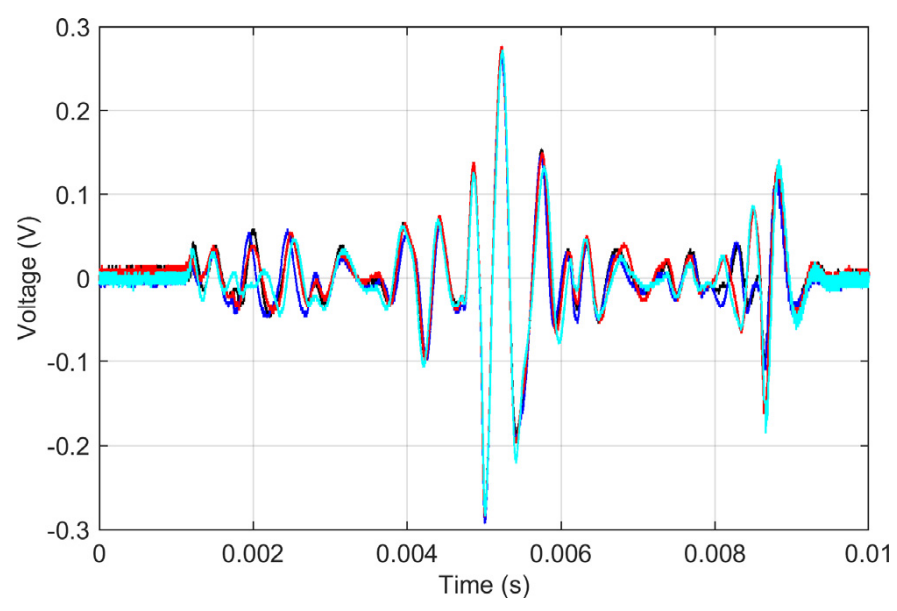

(b)

Figure 3. Typical time-domain waveform of an SMPS at the high-pass filtered LISN output measured as the DSO input signal $y_{1}$ : (a) pattern repetition every $10 \mathrm{~ms}$ with alternated polarity for KT-P25; (b) 4 records, 2 for the positive half-period and 2 for the negative half-period, for LB-P90, showing correspondence for the main pulses but were different in terms of oscillation frequency and damping.

The AC-coupled waveforms shown in Figure $3 \mathrm{~b}$ highlight a fundamental problem of the uniformity and stability of SMPS behavior with respect to unsymmetrical emissions: the two $10 \mathrm{~ms}$ windows of odd and even order show a different time response as if the parasitics and circuit reactive elements were different; the waveforms for two $10 \mathrm{~ms}$ windows of the same type (either both odd or both even) did not perfectly overlap, with some minor discrepancies at some points. Thus, it is understood that the repeatability of unsymmetrical emissions is a relevant point, even in perfectly identical environmental conditions, as it can be over some tens of milliseconds. For this reason, the minimum observation time to derive a reliable frequency spectrum representation must be extended to several $10 \mathrm{~ms}$ periods, in the order of $50-100 \mathrm{~ms}$.

Regarding the distribution of switching pulses and other components along the fundamental period, the acquired signals were processed to scale the $50 \mathrm{~Hz}$ component during post-processing for display purposes, as shown in Figure 4.

The waveforms plotted in Figure 4 show a significant agreement in the position of the main pulse that was caused by the PFC stage. The slight shift observable in some of the curves (where the red and blue curves do not perfectly overlap) was caused by slightly different DSO trigger points.

The peculiar phenomenon occurring in the White SMPS (LW) in large-load conditions (Figure 4a) is shown enlarged in Figure 5, where slightly more than 28 high-frequency switching periods repeated in a timespan of $0.5 \mathrm{~ms}$, indicating a switching frequency of about $56 \mathrm{kHz}$.

\subsection{Basic Post-Processing Methods for the Analysis of Spectral Characteristics}

Spectral characteristics of emissions may be evaluated by either directly measuring in the frequency domain or by applying a time-to-frequency transformation to time-domain data, such as STFT, or alternative approaches, such as wavelet transform and empirical mode decomposition [18]. For simplicity and generality, STFT is applied in the following to the same time-domain data that were anticipated in Section 3.1 and then reported in Section 4.1. 


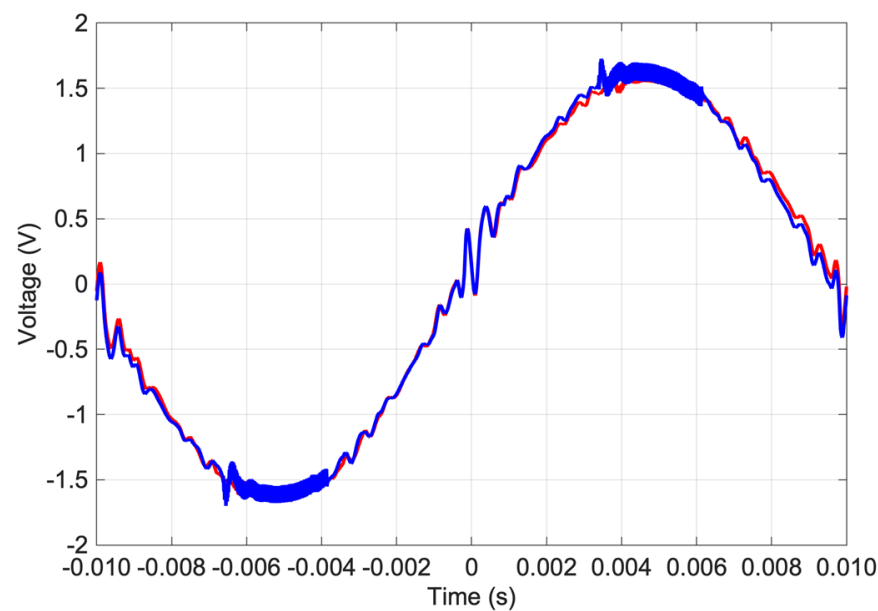

(a)

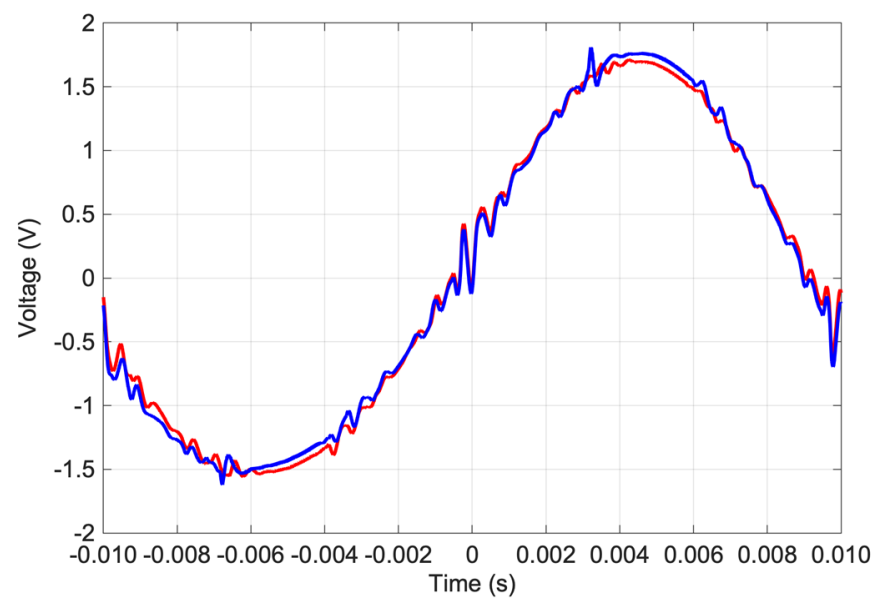

(c)

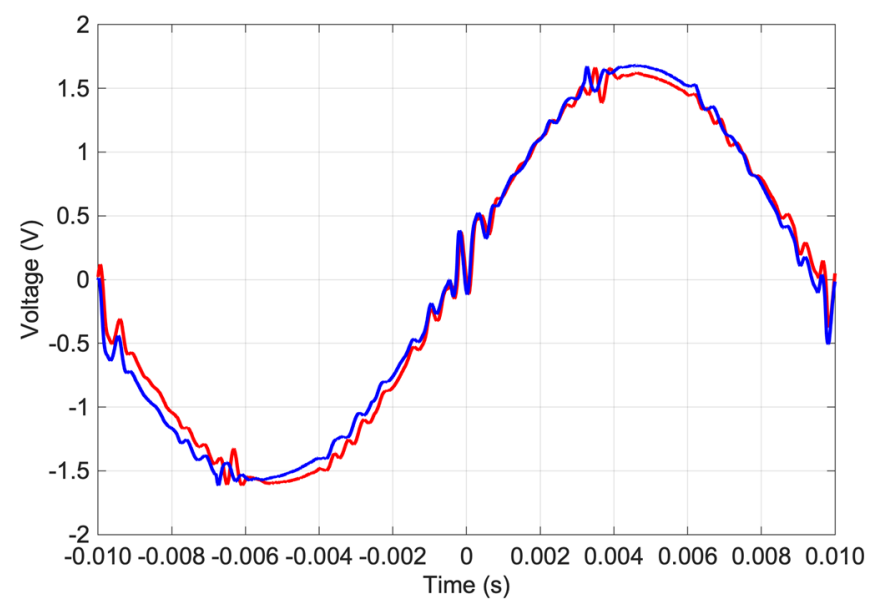

(e)

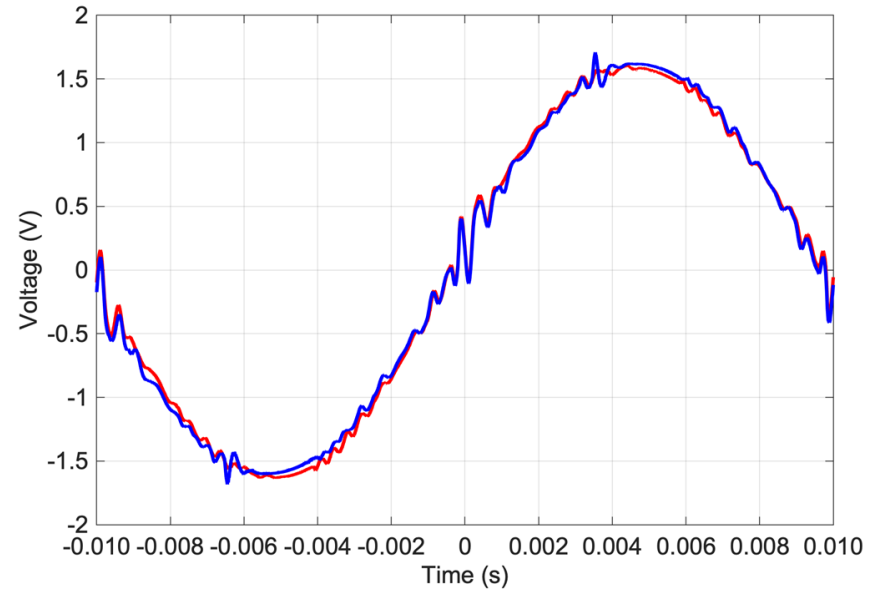

(b)

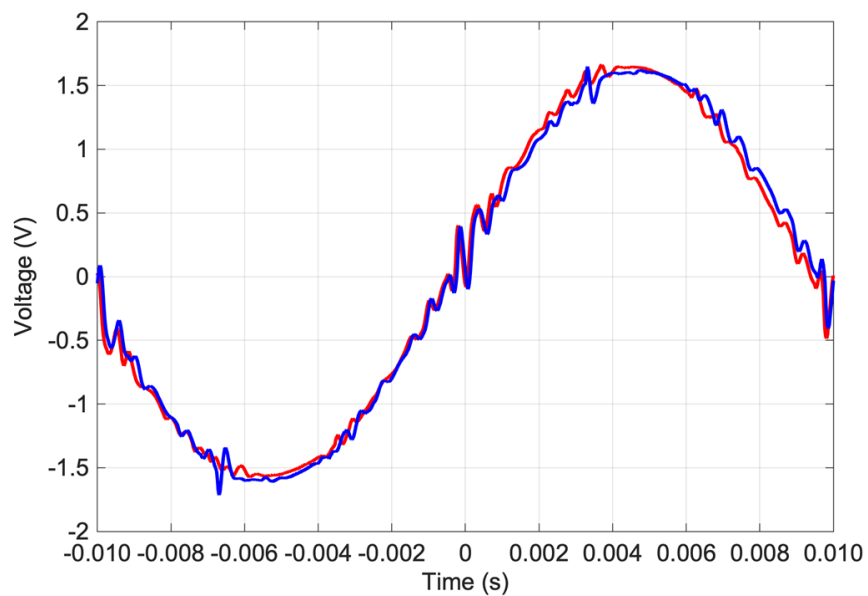

(d)

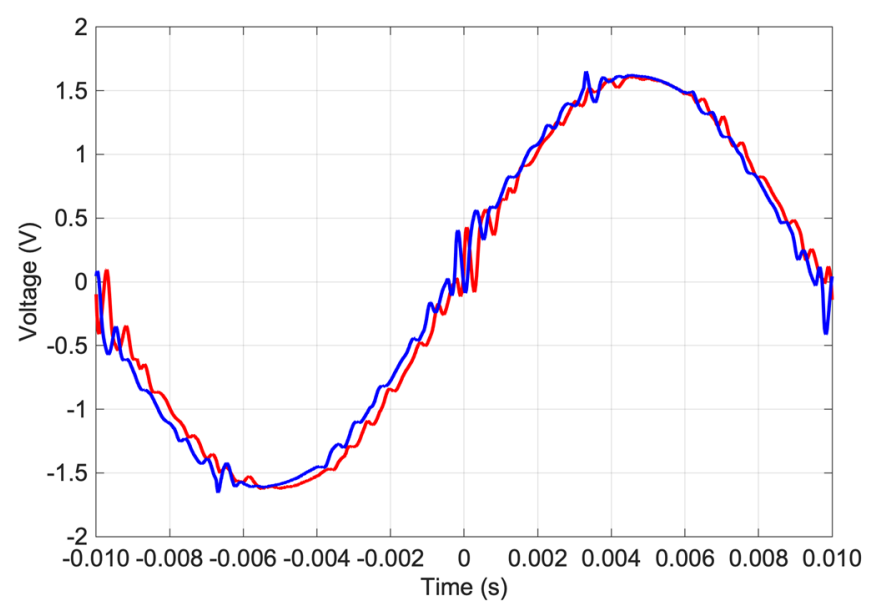

(f)

Figure 4. Time-domain waveforms for $25 \%$ (red) and $90 \%$ (blue) of the load with the $50 \mathrm{~Hz}$ fundamental artificially scaled for display purposes: (a) LW, (b) LB, (c) KT, (d) SH, (e) BA and (f) KA.

As discussed in [17], the various STFT options and parameters represent a source of uncertainty for the spectral amplitude estimate: the approach followed here is that of using a flat-top window that maximized amplitude reading at the expense of a slightly reduced frequency resolution. For ease of interpretation, the base frequency resolution $\Delta f$ was chosen to be equal to $100 \mathrm{~Hz}$, leading to a transformation window $T=10 \mathrm{~ms}$. 
Shorter windows maximize the amplitude estimate for impulsive components that are time localized (short time support), as shown in the sketch of Figure 6, at the expense of a larger variability while sweeping the entire record [17]. Such variability makes it compulsory to undertake max-hold post-processing of the STFT results calculated using such a shorter time window in order to remove the window-to-window variability and produce more stable and consistent estimates. In addition, despite the use of the flat-top smoothing window, the use of a short window length caused larger spectral leakage since the cutting of signal windows often occurs at positions where the signal amplitude is significant and cannot be optimized by looking for points with almost null amplitude (such those intervals between main pulses). Nevertheless, as shown in Figure 6b, short well-positioned time windows can be used to identify transient emissions that become diluted and result in lower amplitude when analyzed with the longer, although more stable, $10 \mathrm{~ms}$ window. Such short-duration emissions may be relevant as a disturbance to, e.g., data transmission protocols (e.g., a serial data line) whose clock and data transmission rate is much faster than the window rather than configuring as a classical disturbance over tens or hundreds of milliseconds (as specified by CISPR and exemplified by the use of quasi-peak detector in frequency domain).

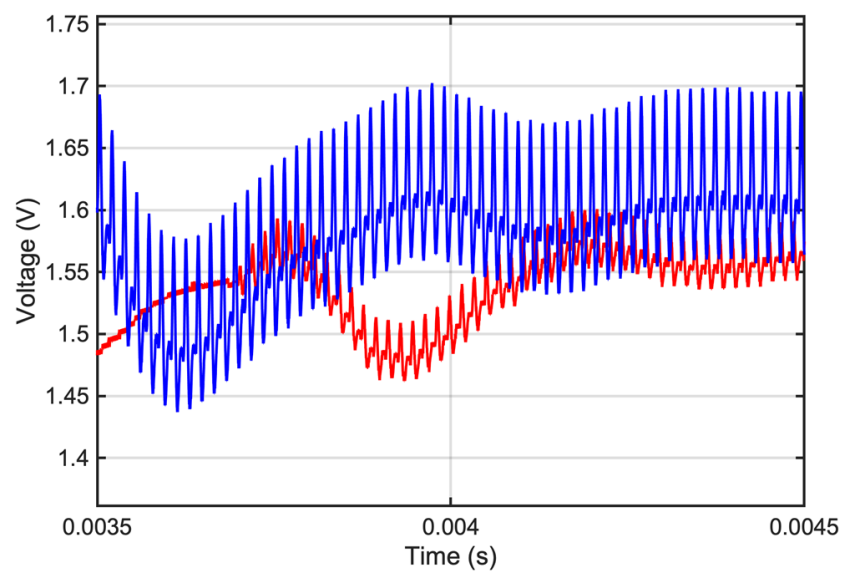

Figure 5. Zoom of the peculiar phenomenon occurring at the minima and maxima of the mains voltage waveform for the LW SMPS (see Figure 4a).

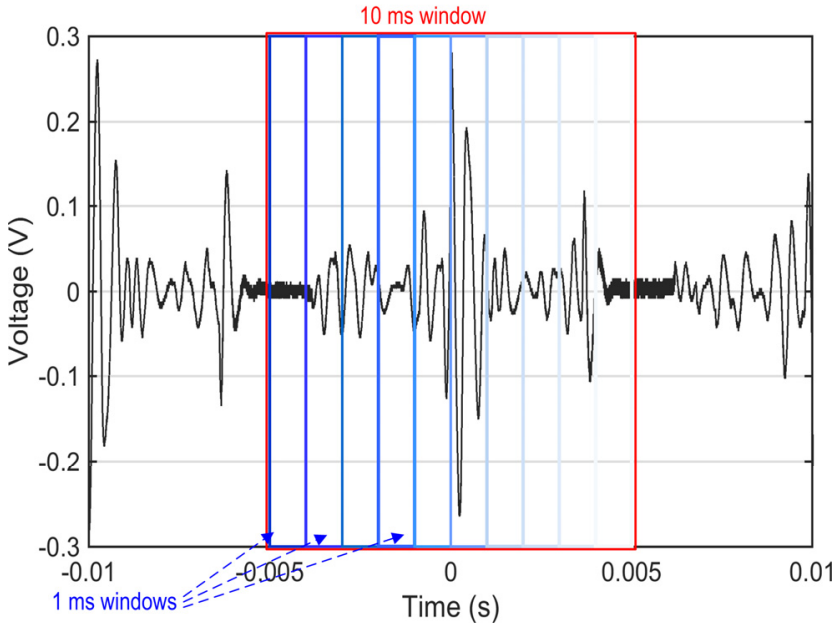

(a)

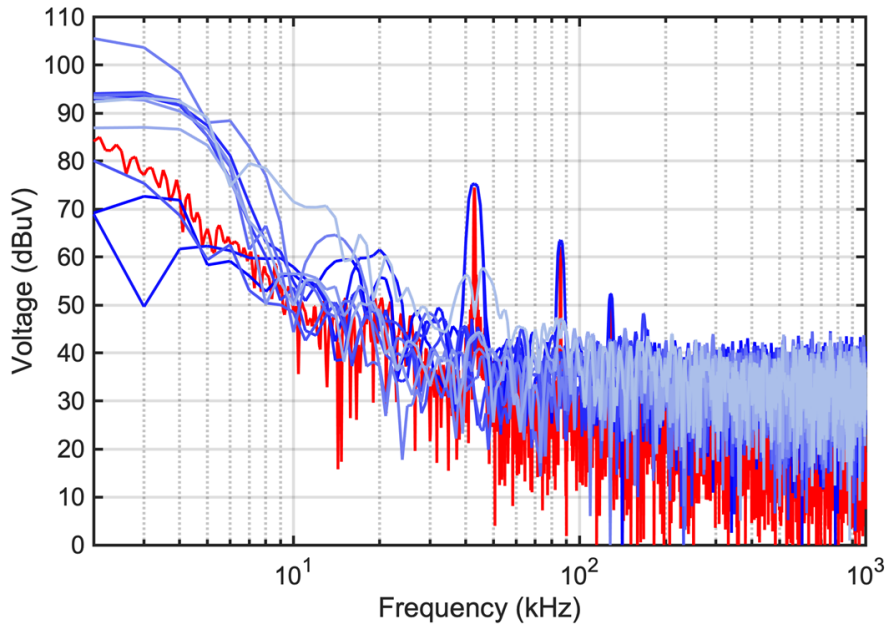

(b)

Figure 6. (a) Arrangement of STFT windows for long (red) and short (blue) window choices ( $T=10 \mathrm{~ms}$ and $1 \mathrm{~ms}$, respectively) and (b) the resulting spectra. 
The equivalence between the STFT spectra and the EMI receiver frequency scans was not immediate, although there was an apparent correspondence between the frequency resolution $\Delta f$ for the former and RBW for the latter. The reason was that, although the choice may be made such that $\Delta f=\mathrm{RBW}$, the EMI receiver has one degree of freedom in almost independently selecting the dwell time (DT), that is, the time interval used to carry out the reading at each frequency bin under the selected aperture represented by the RBW value. Instead, the STFT resolution $\Delta f$ is uniquely related to the transformation window, which is the amount of time over which the "observation" of the signal is carried out. This degree of freedom does not come for free and, as a consequence, an EMI receiver scan may last much longer than an STFT operation.

Seen the other way round, the EMI receiver reading at each frequency bin never uses the "same" signal, but "similar" occurrences of the input signal $y_{1}$ coming from the LISN; the degree of similarity is influenced by the stationarity characteristics of the signal itself. Assuming that the variability of the signal frame after frame is not so significant (see, e.g., Figure $3 b$ ), a limited amount of dwell time is necessary to collect a reliable representation of spectral occurrences at each given frequency bin.

As already observed, a minimum dwell time must be ensured for the signal to pass through all its transient components and repeat itself at the next base period (in principle, $20 \mathrm{~ms}$, but reducible to $10 \mathrm{~ms}$ with some approximation). Such a dwell time ensures consistent measurement of spectral amplitude, increasing the estimate toward the expected maximum amplitude (incoming values at each frequency bin are cumulated with a maxhold peak detector).

However, long waiting times will increase the probability of capturing less frequent transient emissions, which have been observed for some SMPSs, especially at the largest tested loading (90\%). The side effect is a "noisy" spectrum profile, affected by spikes here and there, looking like narrowband emissions because they occur at single frequency bins while sweeping, but are not repeatable, in that they will occur at different frequency bins if the scan is repeated.

The effects of different dwell-time values below and above the reference $10 \mathrm{~ms}$ interval are shown in Figure 7, together with the mentioned spikes, which was particularly evident for the LB-P90 scenario (Figure 7a), but also the SH-P90 case (Figure 7c). 


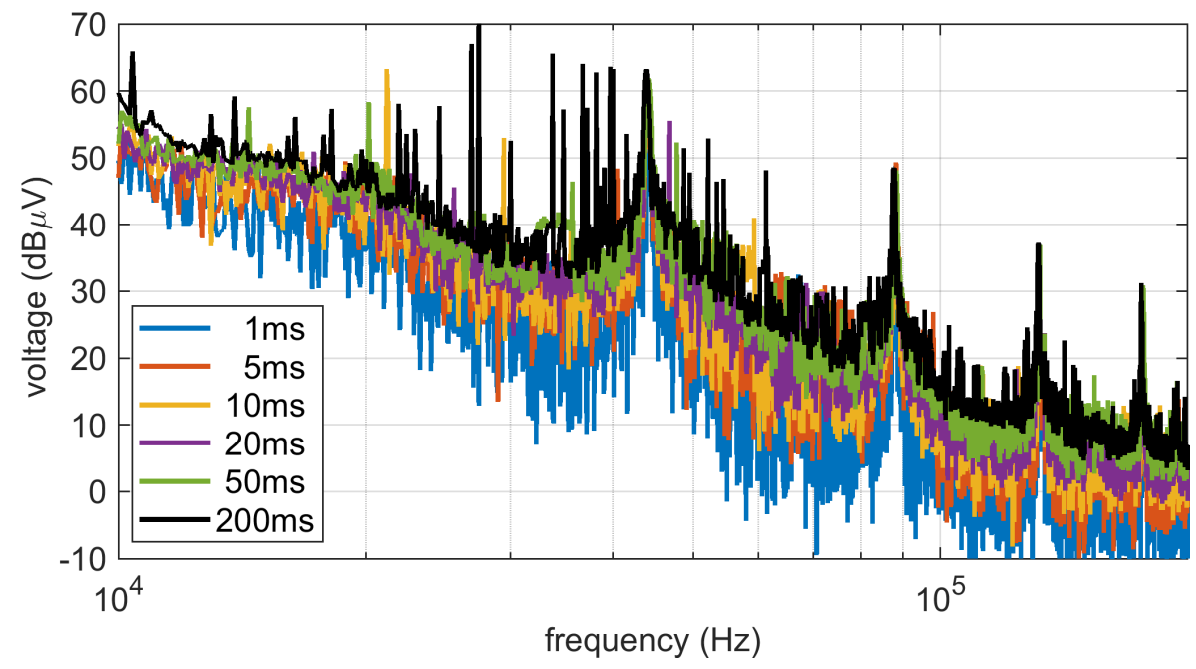

(a)

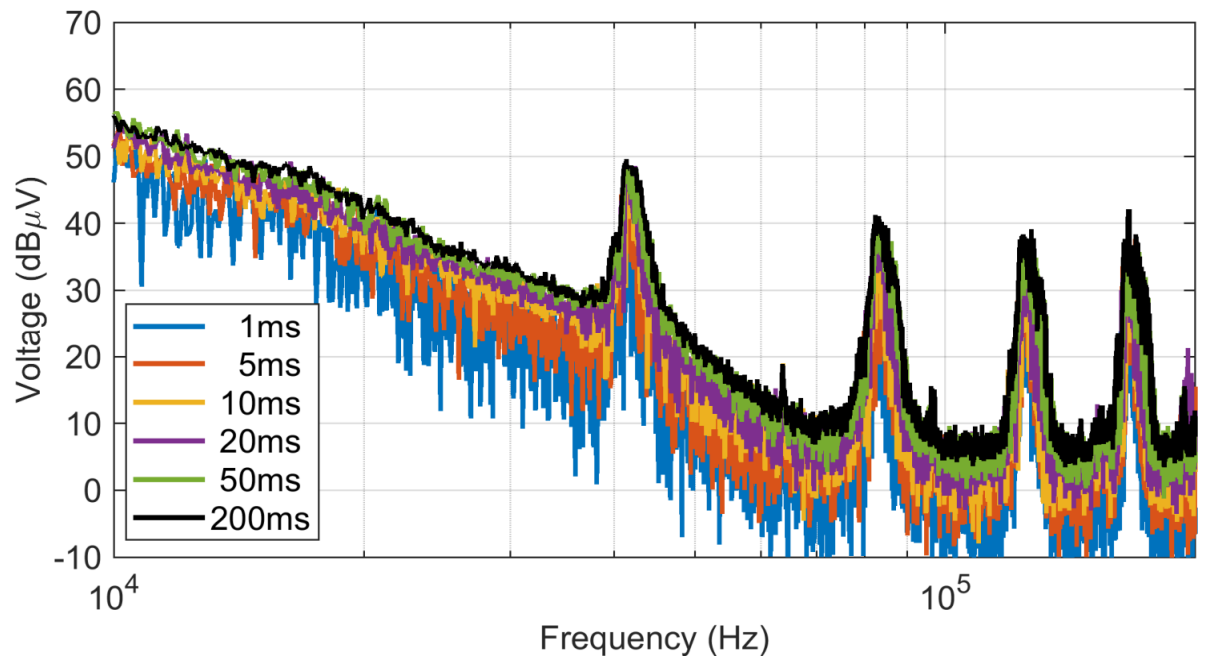

(b)

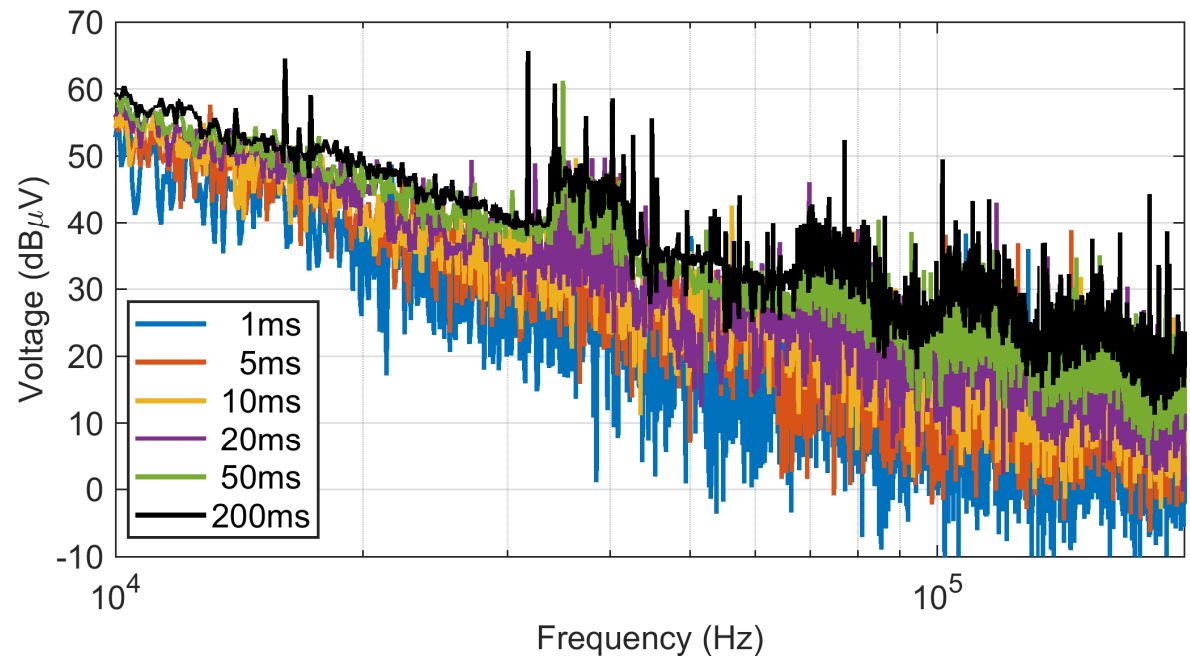

(c)

Figure 7. Effect of the dwell time on the EMI receiver measured spectra over the 10-150 kHz frequency interval for (a) LB-P90, (b) KT-P90 and (c) SH-P90. 


\section{Unprocessed Reference Data}

As observed in Section 2, data obtained with this measurement setup consist of both time- and frequency-domain vectors, measured by means of DSO and EMI receivers, respectively. It is once more underlined that time-domain data allow for more flexible post-processing, whereas EMI receiver scans are a useful reference for comparison, besides still being endorsed by EMC standards.

The reported results in this section document the observed emissions as completely as possible, allowing users to repeat the discussed processing methods, possibly improving parameters settings and exploring new approaches. Such results, which are available as a separate dataset (described in more detail in Appendix A), consist of the following:

- Time-domain waveforms, where signal details in various operating conditions are shown to provide a first visual assessment of signal characteristics, also to better understand the behavior of the processed data; data were sampled at $10 \mathrm{MHz}$ and low-pass filtered for anti-aliasing exigency at $2 \mathrm{MHz}$, ensuring they are fully valid up to $1 \mathrm{MHz}$.

- $\quad$ EMI receiver scans similarly extending up to $1 \mathrm{MHz}$ and using an RBW value of $200 \mathrm{~Hz}$, which is compatible with requirements of EMC standards for the 9-150 kHz interval; the adopted dwell time was $1 \mathrm{~ms}$.

\subsection{Time-Domain Waveforms}

Excerpts of high-pass-filtered time-domain traces lasting for $20 \mathrm{~ms}$ are shown overlapped for $0 \%, 25 \%$ and $90 \%$ load conditions for each of the six SMPSs, shown in Figure 8, were used to evaluate macroscopic changes at various load conditions. These traces for a total duration of $200 \mathrm{~ms}$ represent the time-domain dataset (in brief, the "TD-dataset").

Two SMPSs were then selected to show the macroscopic repeatability of waveforms in different $20 \mathrm{~ms}$ intervals under the same load conditions. The results are shown in Figure 9, where ten such intervals are plotted overlapped without distinction (all traces are black and an indication of variability is the imperfect overlapping, as highlighted by the broadening of traces).

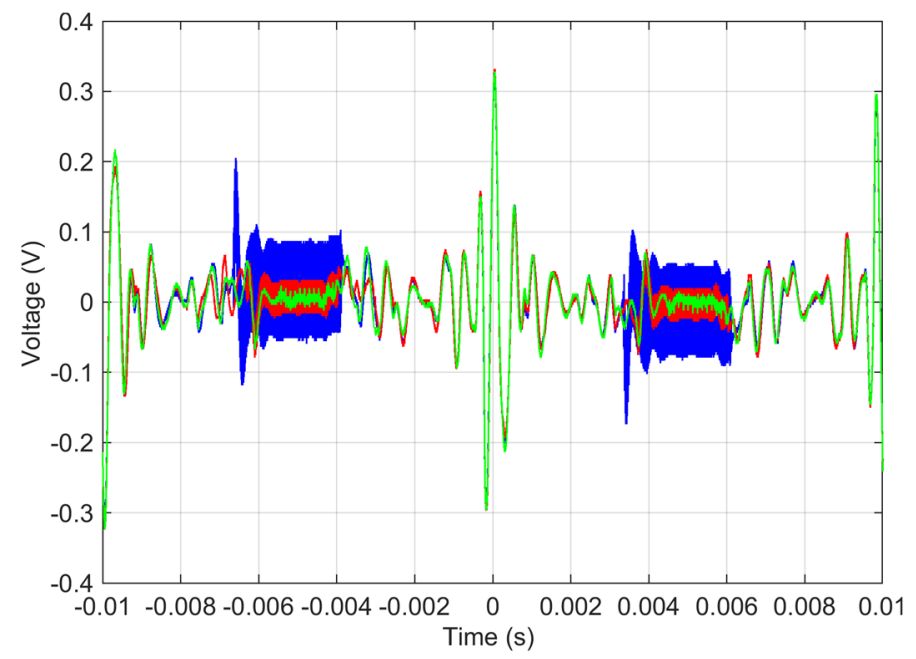

(a)

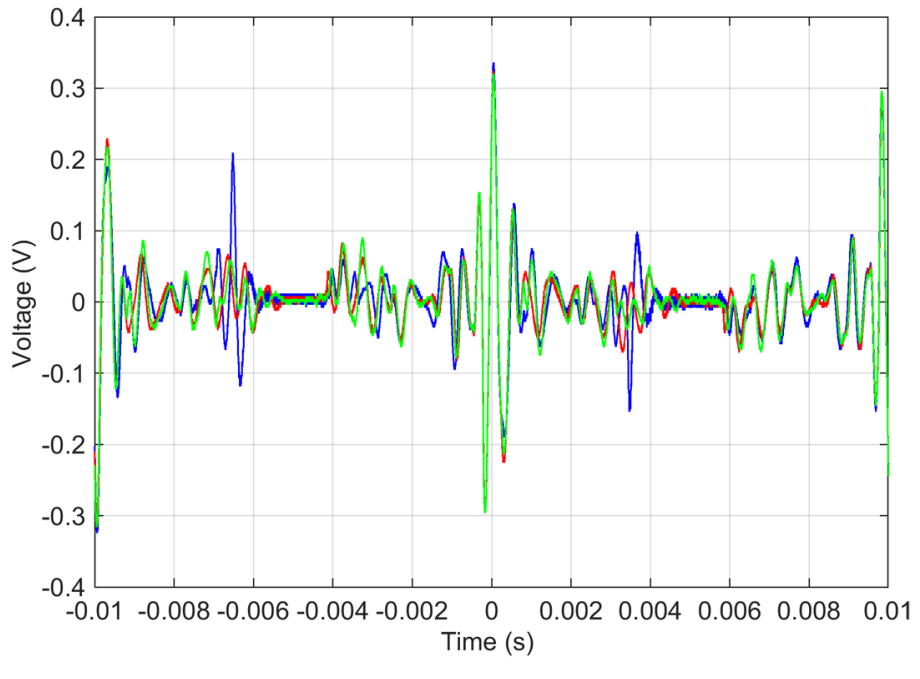

(b)

Figure 8. Cont. 


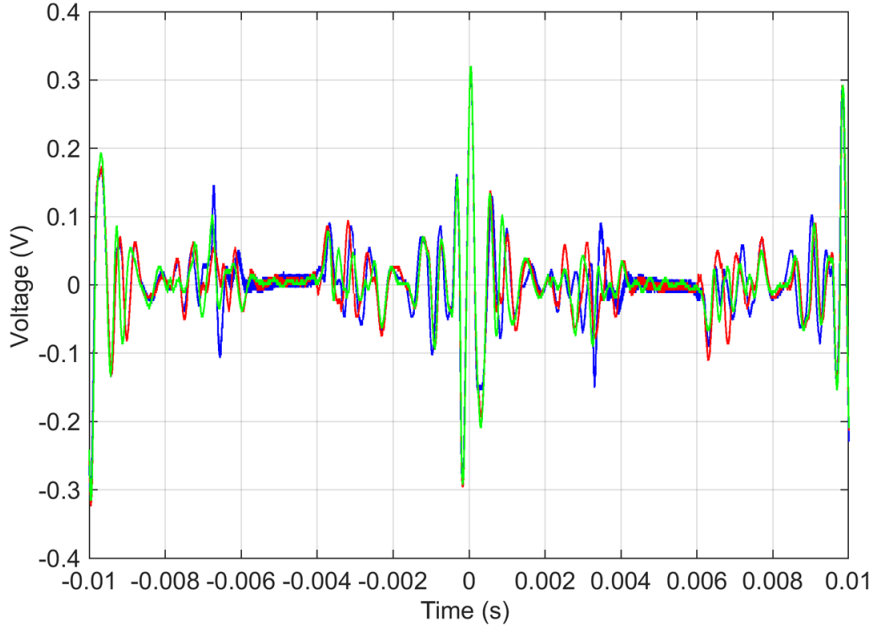

(c)

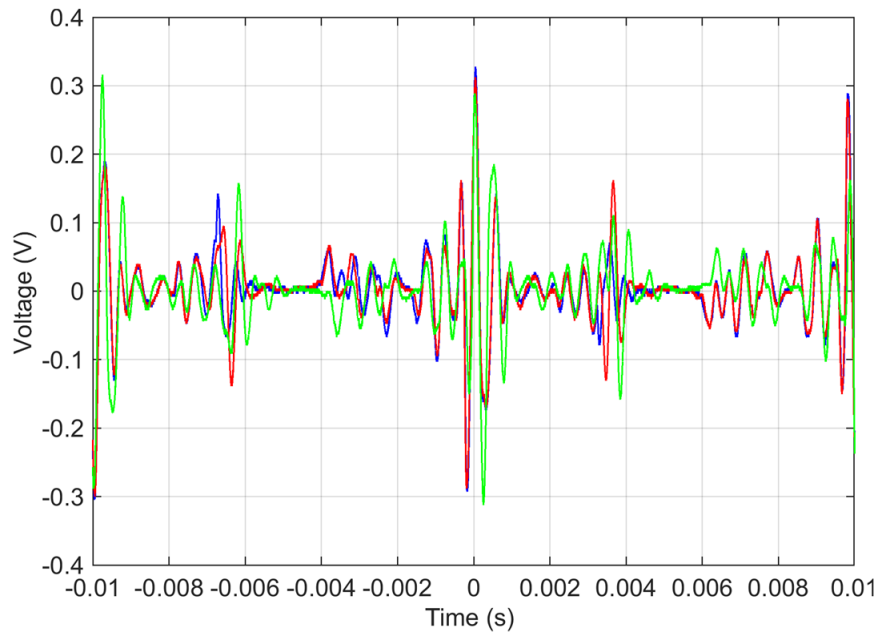

(e)

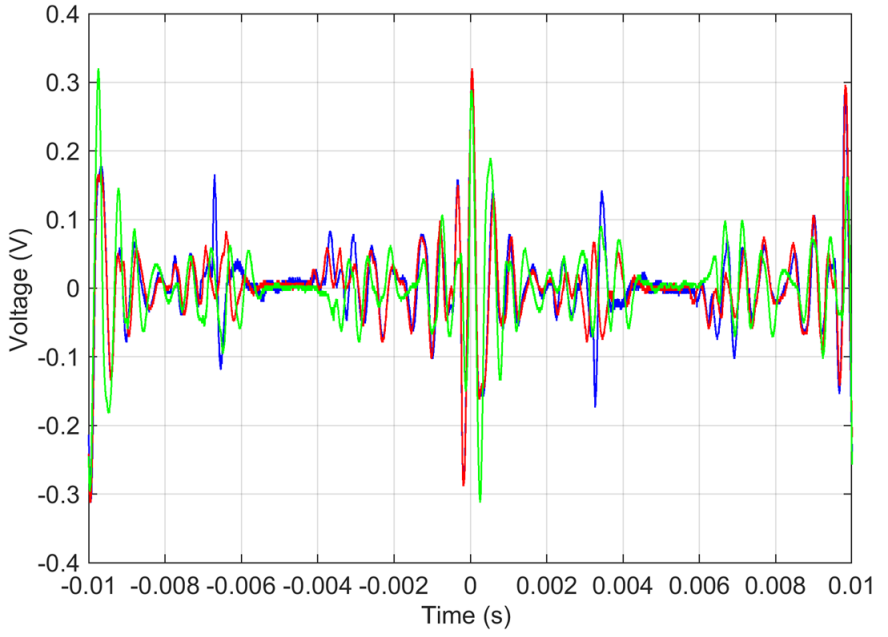

(d)

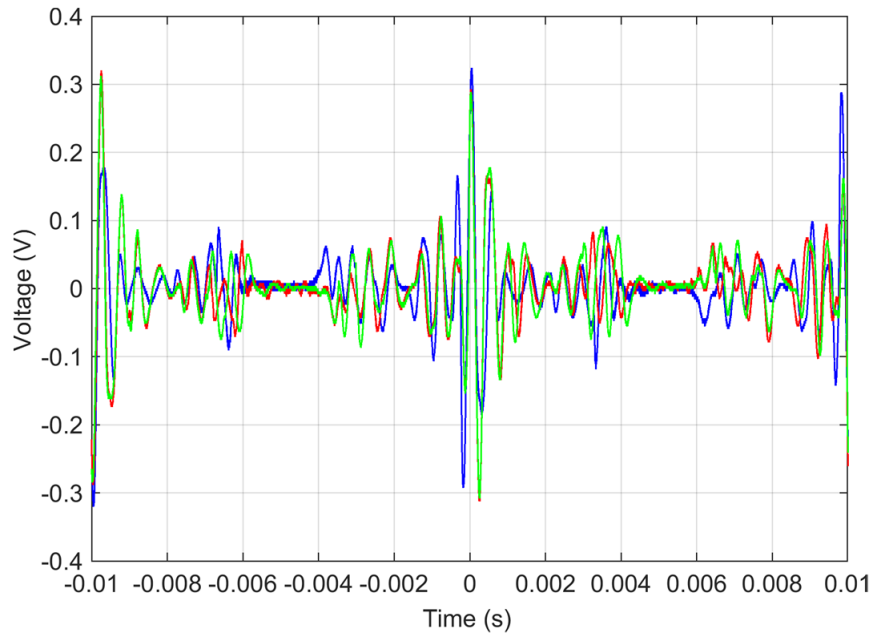

(f)

Figure 8. High-pass filtered time-domain waveforms for no load (green) and $25 \%$ (red) and $90 \%$ (blue) loads: (a) Lacie White, (b) Lacie Black, (c) KTEC, (d) Shenzhen, (e) Balance and (f) Kario.

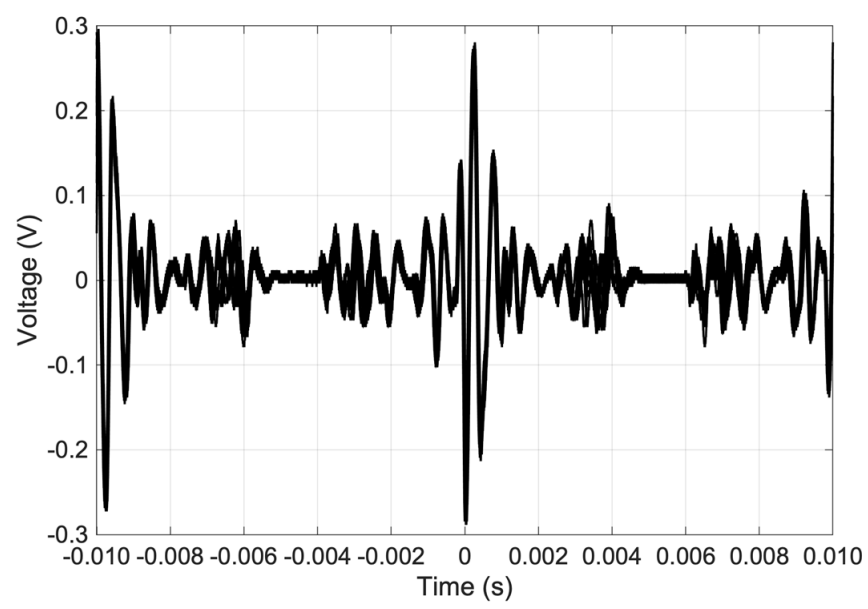

(a)

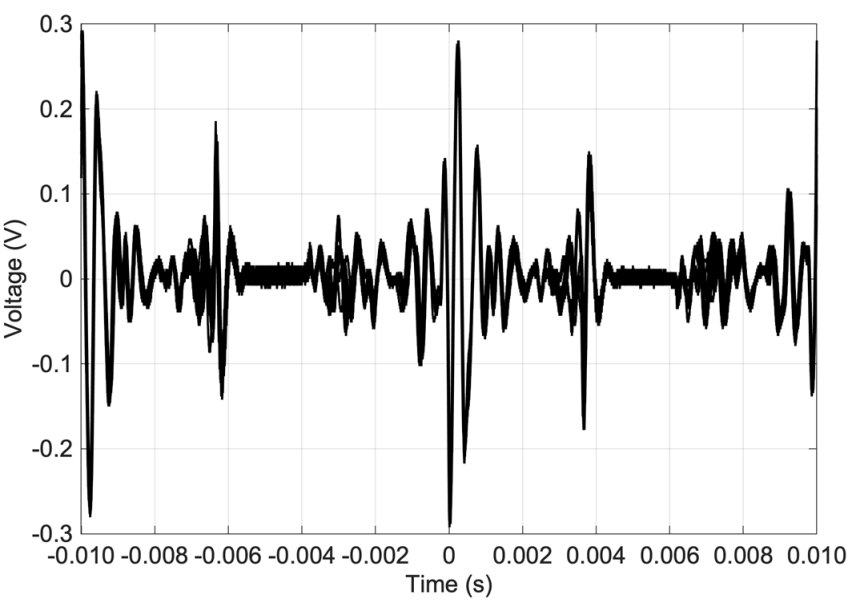

(b)

Figure 9. Cont. 


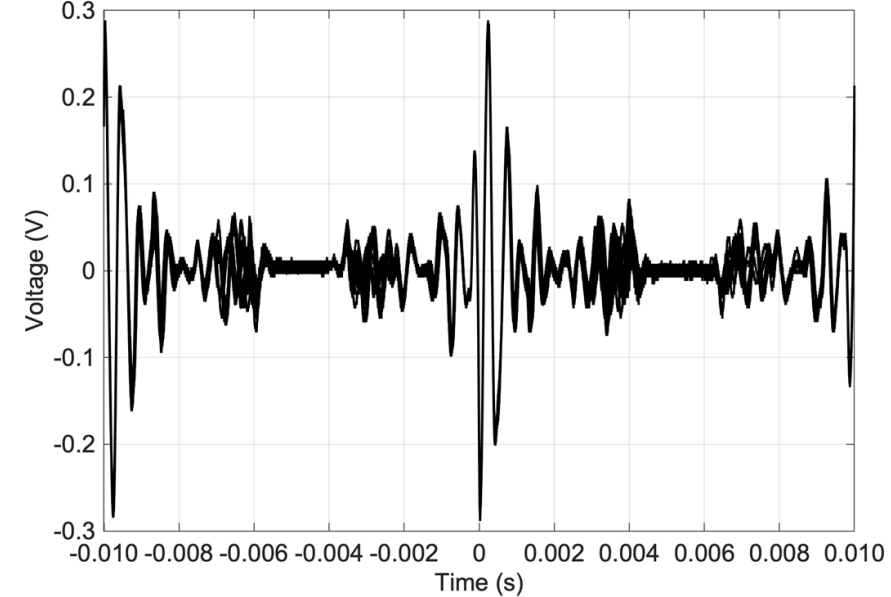

(c)

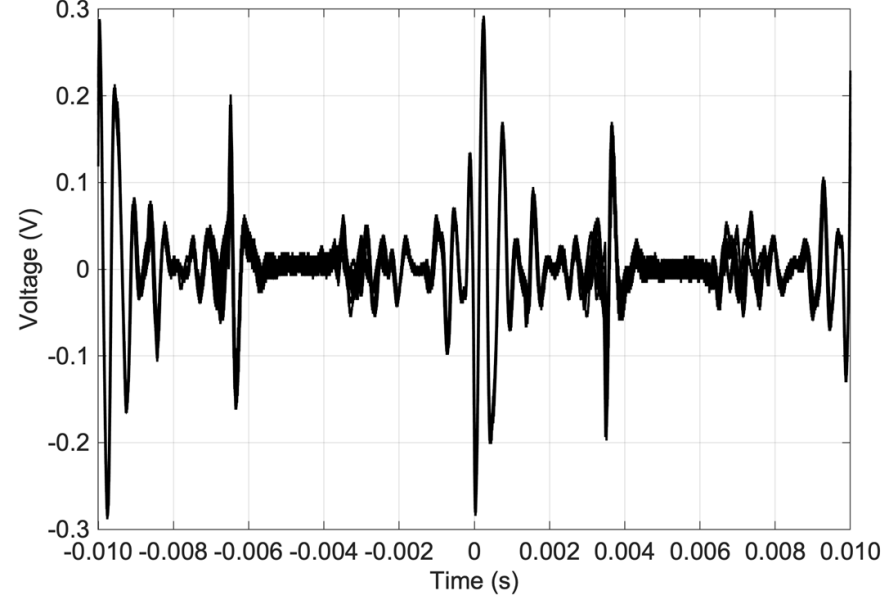

(d)

Figure 9. Ten successive $20 \mathrm{~ms}$ windows overlapped to show time variability of waveform portions: (a) Lacie Black P25, (b) Lacie Black P90, (c) KTEC P25 and (d) KTEC P90.

The overlapped waveforms reported in Figure 9 confirmed that the shape and position of the main pulse were quite stable, whereas oscillations occurring at other time positions during the $50 \mathrm{~Hz}$ period were subject to variability, as evident in particular around $\pm 7 \mathrm{~ms}$ and $\pm 3 \mathrm{~ms}$. This indicated some variability in the medium frequency range, up to what is appreciable by simply inspecting time-domain waveforms, in contrast with the fair stability of the low-frequency components (for which variability of spectrum amplitude is caused by the adopted time window $T$, with such components being of the impulsive type).

\subsection{Frequency-Domain Spectra}

Frequency-domain spectra were acquired with the EMI receiver for the three load conditions of no load ( $0 \%$ ) and $25 \%$ and $90 \%$ of the nominal load. The profiles reported in Figure 10 are available in the FD-dataset, as described in Appendix A. The displayed frequency range in Figure 10 is limited to $150 \mathrm{kHz}$ to improve the visibility.

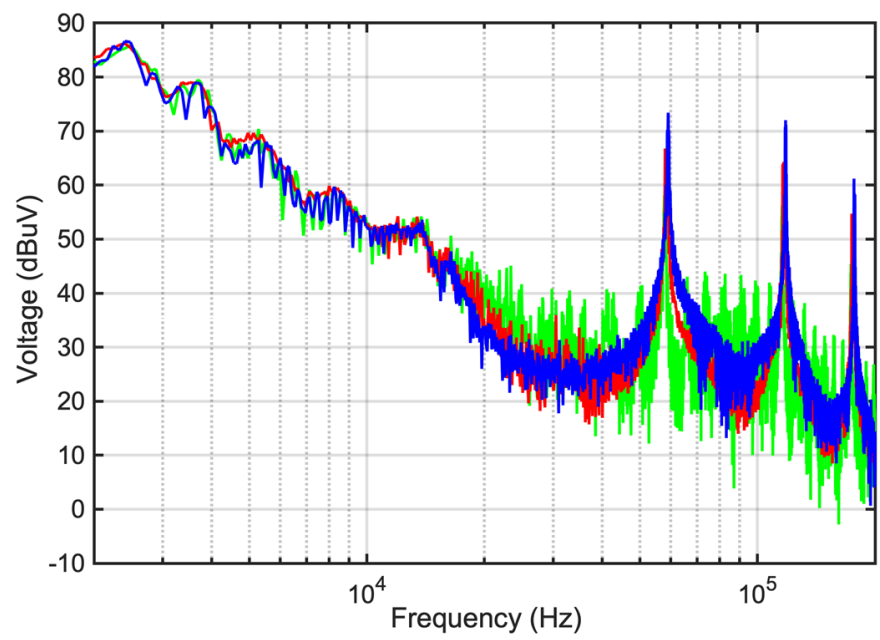

(a)

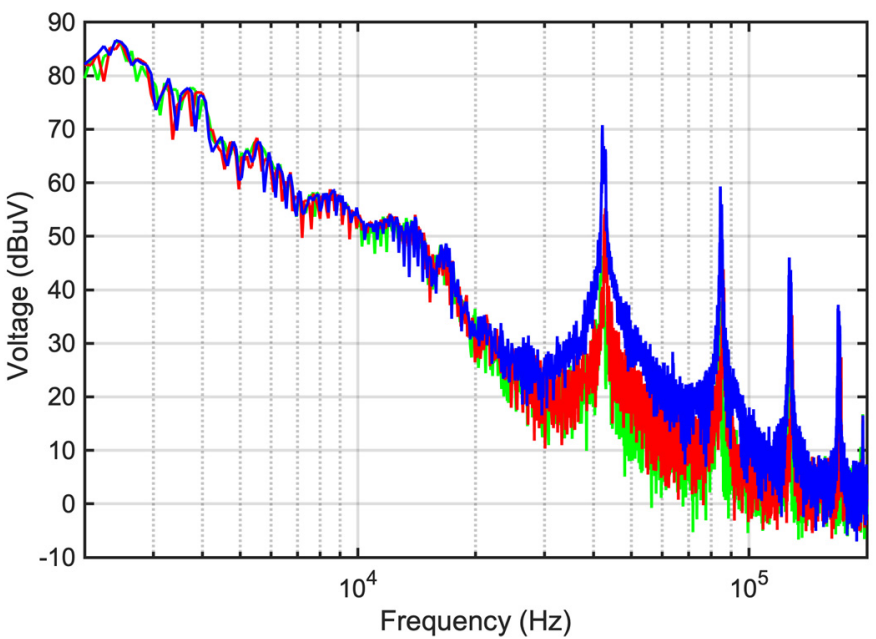

(b)

Figure 10. Cont. 


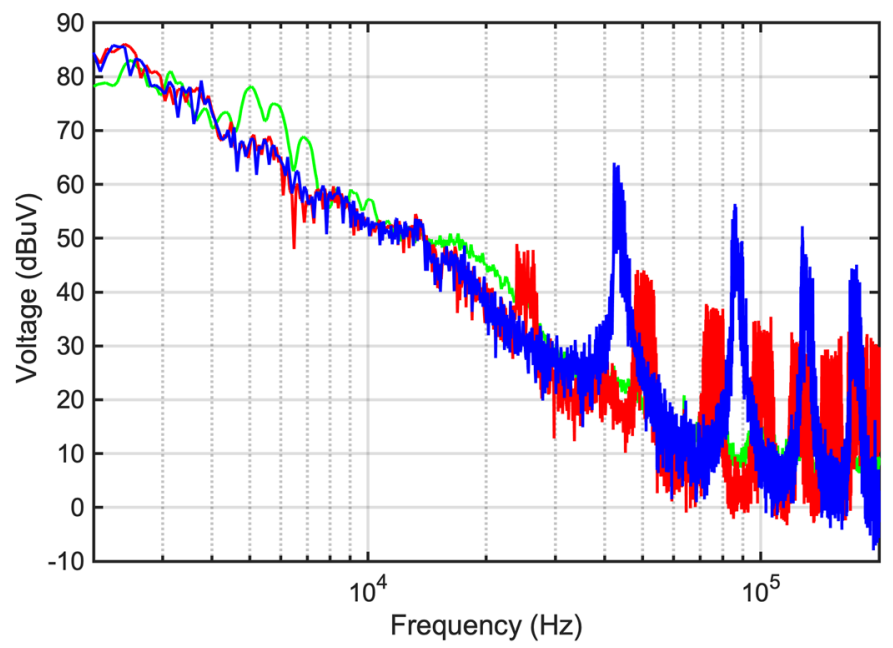

(c)

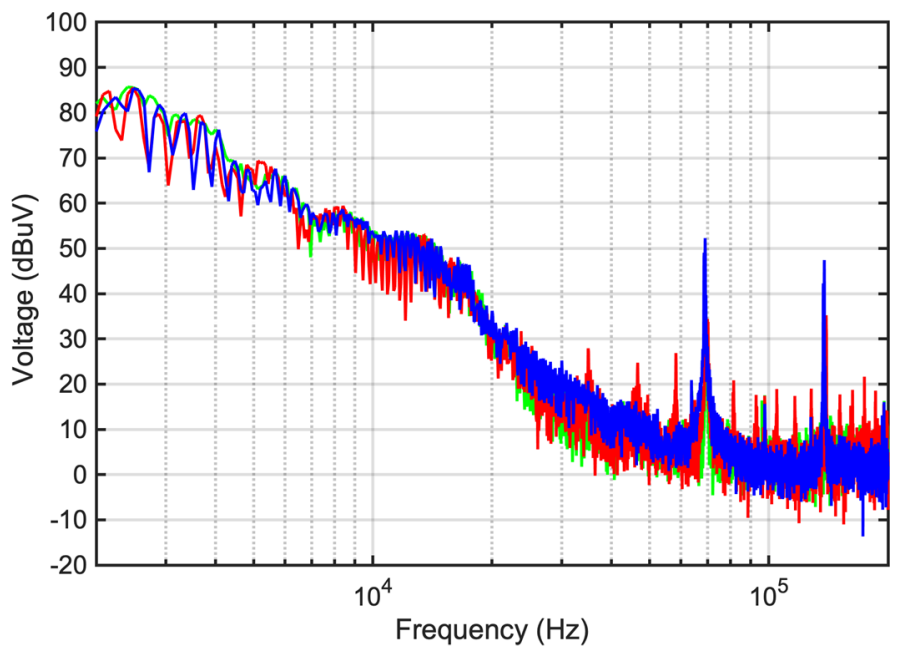

(e)

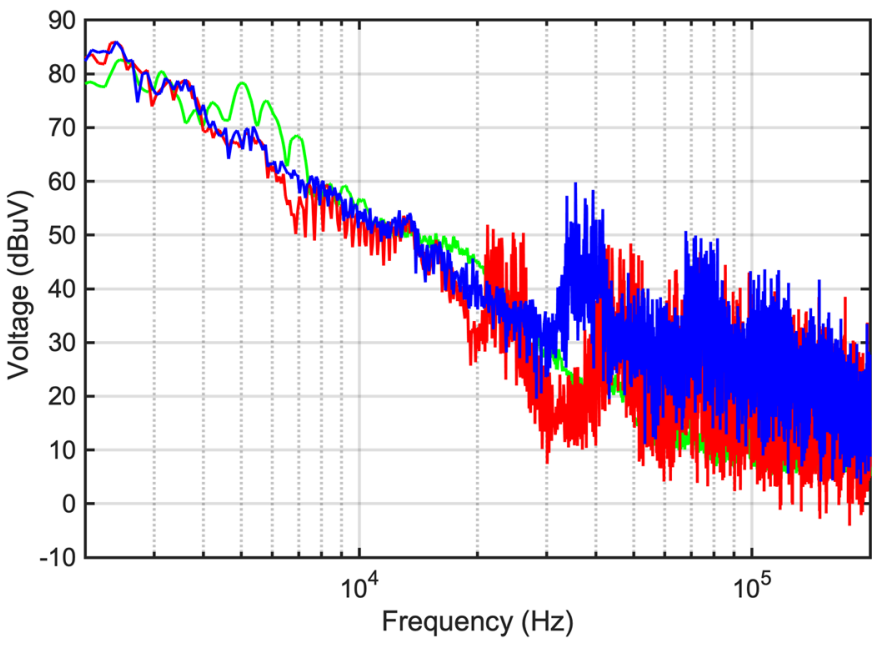

(d)

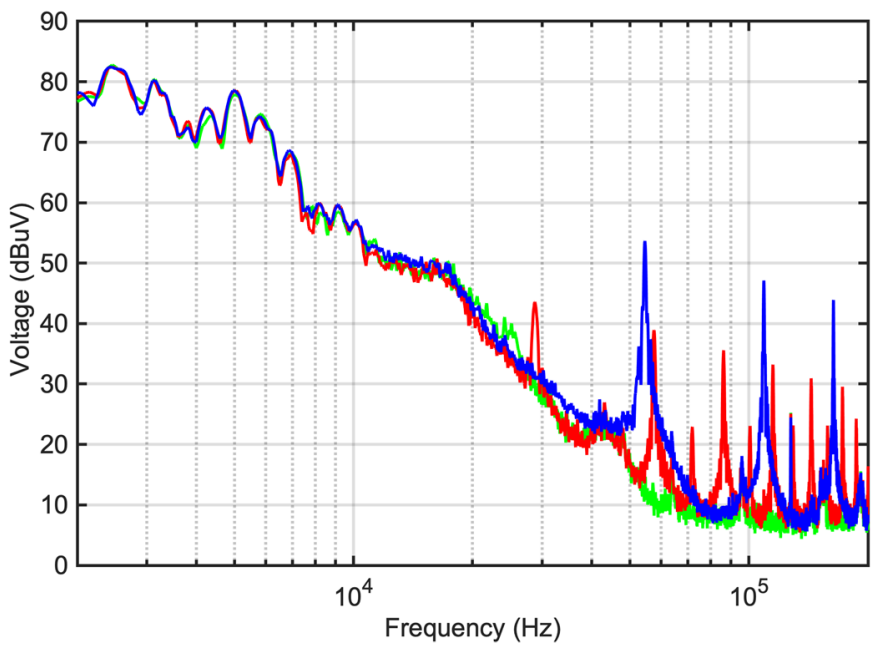

(f)

Figure 10. Frequency-domain spectra for no load (green) and $25 \%$ (red) and $90 \%$ (blue) loads: (a) LW, (b) LB, (c) KT, (d) SH, (e) BA and (f) KA.

The measured spectra provided information on some important points:

- No-load emissions (green curve) could sometimes and for some frequency intervals be larger than curves for the other test conditions such that no load is an important operating condition to include in a test program; see, in particular, Figure 10a, where there was a consistent increase of about $10 \mathrm{~dB}$ above $20 \mathrm{kHz}$, with the appearance of many secondary components.

- In general high-load (90\%) emissions were larger than medium-load (25\%) ones, as was quite evident in almost all cases shown in Figure 10 and, in particular, Figure 10c, where the benefits of random modulation were significantly reduced.

- The $25 \%$ condition, however, presented some secondary emissions that were not visible in the high-load condition, as evident in Figure 10e,f.

\section{Conclusions}

This work discusses the behavior of a large set of switched-mode power supplies regarding unsymmetrical conducted emissions measured by means of an LISN connected to a digital sampling oscilloscope (by means of a cascaded impedance matching stage, high-pass filter and low-pass filter) or directly to an EMI receiver. 
Time-domain and frequency-domain data were considered to highlight the most evident characteristics and to guide aware, not to say correct, post-processing based on short-time Fourier transform, with reference to past work that has thoroughly quantified the effects of parameters such as overlap percentage and the choice of tapering window [17]. More in detail, the effects of deterministic and random modulation were demonstrated, as well as the behaviors at various loading conditions, enabling the selection of adequate measurement and post-processing settings. Some new recommendations for measurement and post-processing are given as follows.

Regarding time-domain measurements, the contribution of the PFC stage to the conducted emissions showed scaling of the $50 \mathrm{~Hz}$ component and superposing the recorded high-pass filtered signal during post-processing. Moreover, it was shown that short wellpositioned time windows could help with individuating transient emissions more effectively than when longer windows were used: in fact, the traces with blue nuance in Figure 10 show a large variability for all transient and broadband components, whereas, for narrowband components, the amplitude was much more stable and corresponded to the red trace obtained with a longer window, equal to the repetition interval of $10 \mathrm{~ms}$. In those cases where transient emissions are of concern (e.g., to evaluate disturbance to some kind of communication system), such shorter time windows should be used.

Regarding the EMI receiver measurements, the effect of the dwell time on the measured spectra was presented. A longer permanence on each spectrum bin to cumulate readings to feed to the used peak detector provided larger and more accurate amplitude estimates; however, this increased the probability of capturing glitches, increasing the presence of outliers and giving more irregular shapes to the spectrum. Optimal values should be longer than the repetition time of $10 \mathrm{~ms}$, in particular, when reading components related to the periodic impulsive transient; values longer than, e.g., $50 \mathrm{~ms}$ significantly increased the presence of outliers.

It is also observed that different load conditions should be taken into account in a test program for SMPS and similar equipment. In fact, although the high-load (90\% case) emission peaks were generally the largest, for some SMPSs, no-load emissions were larger than the $25 \%$ and $90 \%$ loads in specific frequency ranges. Moreover, the $25 \%$ load condition produced some secondary emissions that were not present in the $90 \%$ load, although it was not a strictly necessary test condition to capture the largest emissions. Test conditions should in reality replicate the expected use and operating conditions of the DUT in its final application and it was demonstrated that both low- and high-load conditions were relevant to the conducted emissions and should be replicated.

Supplementary Materials: The following supporting information can be downloaded at: https: / / www.mdpi.com/article/10.3390/electronics11040591/s1. The data presented in this study are available in this article as supplementary files named "TD-dataset" and "FD-dataset".

Author Contributions: The contributions of the two authors L.S. and A.M. to conceptualization, methodology, software, experimental validation, and writing were the same. All authors have read and agreed to the published version of the manuscript.

Funding: This research received no external funding.

Conflicts of Interest: The authors declare no conflict of interest.

\section{Appendix A}

The paper is accompanied by a dataset whose data were previewed in Section 4 and that are organized as follows.

A dataset "TD-dataset" of time-domain waveforms recorded for the six SMPSs using the 8-bit DSO, sampling at $10 \mathrm{MHz}$ for a duration of $200 \mathrm{~ms}$ (2 million samples for each waveform; the tested loading conditions were $0 \%, 25 \%$ and $90 \%$ ). Files are in CSV (commaseparated value) format: one file is the header (not strictly necessary) and the other with 
the word "wfm" is the array of numeric values (readily available without any conversion factor and with an implicit sample time of $100 \mathrm{~ns}$ ).

A dataset "FD-dataset" of frequency-domain spectra measured with an EMI receiver set to RBW $=200 \mathrm{~Hz}$ and dwell time of $1 \mathrm{~ms}$; the scanned frequency interval was $2 \mathrm{kHz}$ to $1 \mathrm{MHz}$ with several samples compatible with the internal EMI receiver memory. Files are in ASCII format with extension.dat; in each file there is a 25-line header and data begin at line 26 with pairs of values providing the frequency in $\mathrm{Hz}$ and amplitude in $\mathrm{dB} \mu \mathrm{V}$; the file then has some lines at the end of the numeric values that are unnecessary and can be discarded.

Datasets are provided in two zip files containing the data files named with the following scheme:

- $\quad$ Name of SMPS: BALANCE, BLACK, KARIO, KTEC, SHENZEN, WHITE;

- Load level: 0A (no load), P25 (25\% of nominal load) and P90 (90\% of nominal load).

\section{References}

1. IEC 61000-3-2; Electromagnetic Compatibility (EMC)—Part 3-2: Limits—Limits for Harmonic Current Emissions (Equipment Input Current $\leq 16$ A Per Phase). IEC: Geneva, Switzerland, 2019.

2. EN 55011; Industrial, Scientific and Medical Equipment—Radio-Frequency Disturbance Characteristics—Limits and Methods of Measurement. CENELEC: Brussels, Belgium, 2021.

3. EN 55032; Electromagnetic Compatibility of Multimedia Equipment-Emission Requirements. CENELEC: Brussels, Belgium, 2020.

4. Thomas, D. Conducted emissions in distribution systems (1 kHz-1 MHz). IEEE Electromagn. Compat. Mag. 2013, 2, 101-104. [CrossRef]

5. Larsson, E.A.; Bollen, M.H.; Wahlberg, M.G.; Lundmark, C.M.; Ronnberg, S.K. Measurements of high-frequency (2-150 kHz) distortion in low-voltage networks. IEEE Trans. Power Del. 2010, 25, 1749-1757. [CrossRef]

6. Frey, D.; Schanen, J.; Quintana, S.; Bollen, M.; Conrath, C. Study of high frequency harmonics propagation in industrial networks. In Proceedings of the International Symposium on Electromagnetic Compatibility-EMC EUROPE, Rome, Italy, 17-21 September 2012. [CrossRef]

7. Bartak, G.F.; Abart, A. EMI in the frequency range $2 \mathrm{kHz}-150 \mathrm{kHz}$. In Proceedings of the CIRED 22nd International Conference on Electricity Distribution, Stockholm, Sweden, 10-13 June 2013.

8. Klatt, M.; Meyer, J.; Schegner, P. Comparison of measurement methods for the frequency range of $2 \mathrm{kHz}$ to $150 \mathrm{kHz}$. In Proceedings of the 2014 16th International Conference on Harmonics and Quality of Power (ICHQP), Bucharest, Romania, 25-28 May 2014. [CrossRef]

9. Sandrolini, L.; Mariscotti, A. Techniques for the Analysis of Time-Domain Conducted Emissions of SMPS in Smart Grids. In Proceedings of the 2019 IEEE International Conference on Environment and Electrical Engineering and 2019 IEEE Industrial and Commercial Power Systems Europe (EEEIC/I\&CPS Europe), Genova, Italy, 11-14 June 2019. [CrossRef]

10. Ensini, L.; Sandrolini, L.; Thomas, D.W.P.; Sumner, M.; Rose, C. Conducted Emissions on DC Power Grids. In Proceedings of the 2018 International Symposium on Electromagnetic Compatibility (EMC EUROPE), Amsterdam, The Netherlands, 27-30 August 2018. [CrossRef]

11. CISPR 16-1-1; Specification for Radio Disturbance and Immunity Measuring Apparatus and Methods-Part 1-1: Radio Disturbance and Immunity Measuring Apparatus-Measuring Apparatus. CISPR: Geneva, Switzerland, 2019.

12. EN 61000-4-19; Electromagnetic Compatibility (EMC)-Part 4-19: Testing and Measurement Techniques-Test for Immunity to Conducted, Differential Mode Disturbances and Signalling in the Frequency Range $2 \mathrm{kHz}$ to $150 \mathrm{kHz}$ at a.c. Power Ports. CENELEC: Brussels, Belgium, 2014.

13. EN 55014-1; Electromagnetic Compatibility-Requirements for House- Hold Appliances, Electric Tools and Similar ApparatusPart 1: Emission. CENELEC: Brussels, Belgium, 2017.

14. EN 55015; Limits and Methods of Measurement of Radio Disturbance Characteristics of Electrical Lighting and Similar Equipment. CENELEC: Brussels, Belgium, 2014.

15. EN 61000-4-30; Electromagnetic Compatibility—Part 4-30: Testing and Measurement Techniques—Power Quality Measurement Methods. CENELEC: Brussels, Belgium, 2015.

16. EN 50065-1; Signalling on Low-Voltage Electrical Installations in the Frequency Range $3 \mathrm{kHz}$ to $148.5 \mathrm{kHz}-\mathrm{Part} 1$ : General Requirements, Frequency Bands and Electromagnetic Disturbances. CENELEC: Brussels, Belgium, 2012.

17. Sandrolini, L.; Mariscotti, A. Impact of Short-Time Fourier Transform Parameters on the Accuracy of EMI Spectra Estimates in the 2-150 kHz Supraharmonics Interval. Electr. Power Syst. Res. 2021, 195, 107130. [CrossRef]

18. Sandrolini, L.; Mariscotti, A. Signal Transformations for Analysis of Supraharmonic EMI caused by Switched-Mode Power Supplies. Electronics 2020, 9, 2088. [CrossRef]

19. Mariscotti, A.; Sandrolini, L. Variability of EMI Measurement for Switched Mode Power Supplies EMI in the 2-1000 kHz Range. In Proceedings of the IEEE 11th International Workshop on Applied Measurements for Power Systems, Cagliari, Italy, 29 September-1 October 2021. [CrossRef]

20. Brander, T.; Gerfer, A.; Rall, B.; Zenkner, H. Trilogy of Magnetics, 5th ed.; Swiridoff Verlag: Waldenburg, Germany, 2018. 
21. Pressman, A.I.; Billings, K.; Morey, T. Switching Power Supply Design, 3rd ed.; McGraw Hill: New York, NY, USA, 2009.

22. Rice, J.; Gehrke, D.; Segal, M. Understanding Noise-Spreading Techniques and their Effects in Switch-Mode Power Applications, Texas Instruments. 2011. Available online: https://www.ti.com/seclit/ml/slup269/slup269.pdf (accessed on 28 December 2021).

23. Iraheta, A. Further Optimizing EMI with Spread Spectrum, Texas Instruments. March 2021. Available online: https://www.ti. com/lit/pdf/slvaf18 (accessed on 28 December 2021).

24. Curtis, P.; Lee, E. EMI Reduction Technique, Dual Random Spread Spectrum, Texas Instruments. June 2020. Available online: https://www.ti.com/lit/pdf/snva974 (accessed on 28 December 2021).

25. Reduction of Switched Mode Power Supply Electro-Magnetic Emissions in Automotive Cockpit Applications, ON Semiconductor. April 2013. Available online: https:/ / www.onsemi.com/pub/collateral/tnd6032-d.pdf (accessed on 28 December 2021).

26. Wan, L.; Khilnani, A.; Hamid, A.; Grassi, F.; Spadacini, G.; Pignari, S.; Sumner, M.; Thomas, D. Limitations in Applying the Existing LISN Topologies for Low Frequency Conducted Emission Measurements and Possible Solution. In Proceedings of the AsiaPacific International Symposium on Electromagnetic Compatibility (APEMC), Nusa Dua-Bali, Indonesia, 27-30 September 2021. [CrossRef]

27. Streubel, T.; Kattmann, C.; Eisenmann, A.; Rudion, K. Characterization of Supraharmonic Emission from Three Different Electric Vehicle Charging Infrastructures in Time and Frequency Domain. Energies 2022, 15, 394. [CrossRef] 